\title{
Erratum to: miRNAs and sports: tracking training status and potentially confounding diagnoses
}

Anne Hecksteden ${ }^{1 \dagger}$, Petra Leidinger ${ }^{2 \dagger}$, Christina Backes ${ }^{3}$, Stefanie Rheinheimer ${ }^{2}$, Mark Pfeiffer ${ }^{4}$, Alexander Ferrauti ${ }^{5}$, Michael Kellmann ${ }^{5,6}$, Farbod Sedaghat-Hamedani ${ }^{7}$, Benjamin Meder ${ }^{7}$, Eckart Meese ${ }^{2}$, Tim Meyer ${ }^{1}$ and Andreas Keller ${ }^{3^{*}}$ (D)

\section{Erratum to: J Transl Med (2016) 14:219 DOI 10.1186/s12967-016-0974-x}

Unfortunately, the original version of this article [1] contained an error. The name of the author [Farbod Sedaghat] was incorrect, the author's surname should be Sedaghat-Hamedani. The author's name is Farbod Sedaghat-Hamedani.

\begin{abstract}
Author details
${ }^{1}$ Institute of Sports and Preventive Medicine, Saarland University, Saarbrücken, Germany. ${ }^{2}$ Department of Human Genetics, Saarland University, Saarbrücken, Germany. ${ }^{3}$ Chair for Clinical Bioinformatics, Medical Department, Saarland University, Building E2.1, 66125 Saarbrücken, Germany. ${ }^{4}$ Department of Theory and Practice of Sports, Johannes Gutenberg University of Mainz, Mainz, Germany. ${ }^{5}$ Faculty of Sport Science, Ruhr-University Bochum, Bochum, Germany. ${ }^{6}$ School of Human Movement Studies, The University of Queensland, St Lucia, Australia. ${ }^{7}$ Internal Medicine, Heidelberg University, Heidelberg, Germany.
\end{abstract}

The online version of the original article can be found under doi:10.1186/s12967-016-0974-x.

Published online: 13 October 2016

\section{Reference}

1. Hecksteden A, Leidinger P, Backes C, Rheinheimer S, Pfeiffer M, Ferrauti A, Kellmann M, Sedaghat-Hamedani F, Meder B, Meese E, Meyer T, Keller A. miRNAs and sports: tracking training status and potentially confounding diagnoses. J Transl Med. 2016;14:219. doi:10.1186/s12967-016-0974-Х.

\footnotetext{
*Correspondence: andreas.keller@ccb.uni-saarland.de

${ }^{\dagger}$ Anne Hecksteden and Petra Leidinger contributed equally to this work

${ }^{3}$ Chair for Clinical Bioinformatics, Medical Department, Saarland

University, Building E2.1, 66125 Saarbrücken, Germany

Full list of author information is available at the end of the article
} 